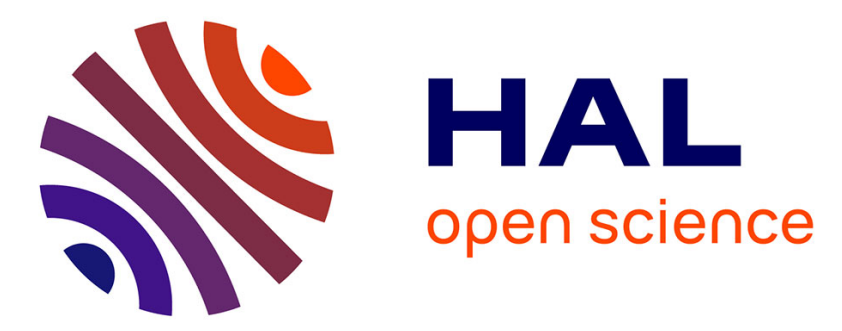

\title{
Generation of optically synchronized pump-signal beams for ultrafast OPCPA via the optical Kerr effect
}

\author{
Christina Alexandridi, Xavier Délen, Frederic Druon, Patrick Georges, Luc
} Martin, François Mathieu, Dimitris Papadopoulos

\section{To cite this version:}

Christina Alexandridi, Xavier Délen, Frederic Druon, Patrick Georges, Luc Martin, et al.. Generation of optically synchronized pump-signal beams for ultrafast OPCPA via the optical Kerr effect. Optics Letters, 2021, 46 (9), pp.2035. 10.1364/OL.425237 . hal-03280230

\section{HAL Id: hal-03280230 \\ https://hal.sorbonne-universite.fr/hal-03280230}

Submitted on 7 Jul 2021

HAL is a multi-disciplinary open access archive for the deposit and dissemination of scientific research documents, whether they are published or not. The documents may come from teaching and research institutions in France or abroad, or from public or private research centers.
L'archive ouverte pluridisciplinaire HAL, est destinée au dépôt et à la diffusion de documents scientifiques de niveau recherche, publiés ou non, émanant des établissements d'enseignement et de recherche français ou étrangers, des laboratoires publics ou privés. 


\title{
Generation of optically synchronized pump-signal beams for ultrafast OPCPA via the optical Kerr effect
}

\author{
Christina Alexandridi ${ }^{1,2}$, Xavier Délen², Frederic Druon ${ }^{2}$, Patrick Georges ${ }^{2}$, Luc \\ MARTIN $^{1}$, François Mathieu ${ }^{1}$, ANd Dimitris Papadopoulos ${ }^{1 *}$ \\ ${ }^{1}$ Laboratoire pour l'Utilisation des Lasers Intenses, C.N.R.S., Ecole Polytechnique, Université Pierre et Marie Curie, 91120 Palaiseau, France \\ ${ }^{2}$ 2Université Paris-Saclay, Institut d'Optique Graduate School, CNRS, Laboratoire Charles Fabry, 91127 Palaiseau, France \\ * Corresponding authors: papadopoulos.d@bureau.luli.polytechnique.fr
}

In recent years, multi-PW laser installations have achieved unprecedented peak powers opening new horizons to laser-matter interaction studies. Ultra broadband and extreme temporal contrast pulses requirements make the optical parametric chirped pulse amplification (OPCPA) in the few-ps regime, the key technology in these systems. To guarantee however a high fidelity output, the OPCPA requires excellent synchronization between the pump and the signal pulses. Here, we propose a new highly versatile architecture for the generation of optically synchronized pumpsignal pairs based on the Kerr shutter effect. We obtained $>550 \mu \mathrm{J}$ pump pulses of 12 ps duration at $532 \mathrm{~nm}$ optically synchronized with a typical ultrashort CPA source at $800 \mathrm{~nm}$. As a proof of principle demonstration, our system has been also used for the amplification of $\sim 20 \mu \mathrm{J}$ ultra-broadband pulses based on an OPCPA setup.

Since the first demonstration almost three decades ago [1], OPCPA has become a key technology of many scientific fields spanning from the attosecond science [2] to strong-field physics [3]. Nowadays, thanks to the unique properties of OPCPAs in the few ps regime, scientists are able to generate ultrashort, high contrast, extreme intense laser pulses that will allow the study of extreme relativistic phenomena. Several PW-class laser installations incorporating the OPCPA technology are under construction throughout the globe [4] reaching today impressive performances on the $10 \mathrm{PW}$ level [5].

However, despite its success, OPCPA remains a demanding technique mainly due to the challenging task of synchronizing its pump and signal pulses. In order to secure high spectral stability and temporal contrast of the final amplified signal, a sub-100 fs synchronization is needed. Different techniques have been tested throughout the years using electronic and optical approaches not free of complications regarding the long term reliability $[6,7]$. Today, the most widely used methods are 1) the generation of both signal and pump pulses from an extremely large bandwidth Ti:Sa oscillator [8-10] and 2) the use of ps Ybbased oscillators where after different types of amplification, part of their output produces the pump while the rest produces the seed through white light generation [11, 12]. The main drawbacks of both methods originate from their specificity regarding both the targeted performances and the involved equipment. In this Letter we propose a new, highly versatile approach for the generation of optically synchronized pump-signal pairs, compatible with high fidelity, high contrast ps OPCPA sources.

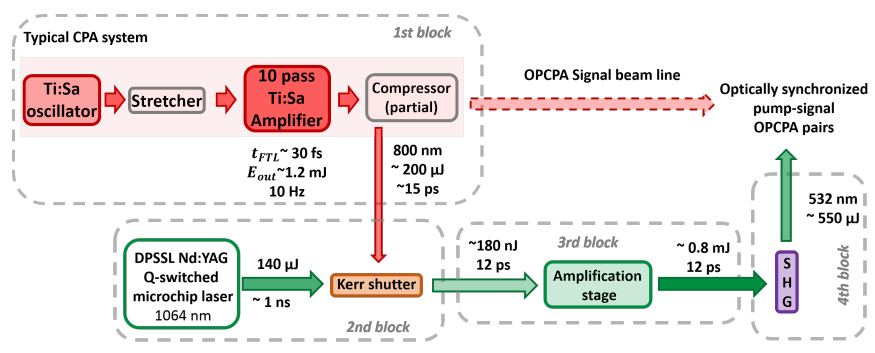

Fig. 1. Overview of the experimental scheme for the generation of the synchronised pump pulses. It consist of four blocks: the CPA line that generates the control beam, the $1 \mu \mathrm{m}$ ns oscillator and the kerr shutter, a Nd: $\mathrm{YVO}_{4}$ based amplifier and the frequency doubling crystal. SHG: second harmonic generation. DPSSL: diode-pumped solid-state laser.

The key element of this new architecture, is a novel technique for optically gating ultra-short laser signals based on the optical Kerr effect, combined with high gain diode pumped amplification modules. The Kerr shutter is a well-established technique used for ultrafast non-linear (NL) gating in the domain of fiber based telecommunications [13] and high-speed imaging [14]. Here exploited under a different context it could provide a robust solution for the generation of ps pulses for the injection of high gain $1 \mu \mathrm{m}$ amplifiers practically perfectly synchronized with any chirped pulse amplification (CPA) system.

Using a fraction of an intense $800 \mathrm{~nm}$ beam, called hereafter 
the control beam, we can drive a NL gate based on the Kerr shutter operation principle so that the polarization state of another ns beam at $1 \mu \mathrm{m}$ is instantaneously modified over a small temporal fraction of the initial pulse. Choosing accordingly the pulse duration of the control pulses and the energy of the 1 ns oscillator, can lead to the generation of the required pump pulses that perfectly satisfy the synchronization needs of ultrafast OPCPA beam lines. A complete schematic of the setup is provided in Fig. 1. Our system can be divided into four main blocks: 1) A homemade CPA line that provides high intensity pulses used to drive the optical NL gate, 2) The NL gate itself, 3) the $1 \mu \mathrm{m}$ amplification stage and 4) the frequency doubling stage of the amplified pulses.

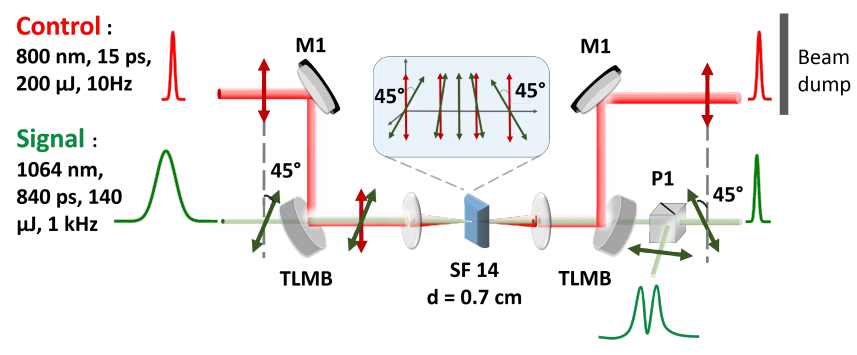

Fig. 2. Experimental set up of the Kerr shutter. High Energy Ti:Sapphire Femtosecond Mirrors (TLMB): highly reflective mirrors for $800 \mathrm{~nm}$ at $45^{\circ}$, partially transparent at $1064 \mathrm{~nm}$. M1: silver mirrors, P1: polarizer.

The first block is a typical Ti:Sa based CPA system. After stretching the oscillator pulses ( $\sim 30 \mathrm{fs}$ at $800 \mathrm{~nm})$ to $\sim 150 \mathrm{ps}$ using an Offner stretcher, they are amplified in a multipass Ti:Sa amplifier up to $1.2 \mathrm{~mJ}$ at $10 \mathrm{~Hz}$. A diffraction grating compressor then partially compresses the pulses down to a duration estimated around 15 ps and a fraction of $200 \mu \mathrm{J}$ of their energy serves as the control beam.

The second block consists of the NL gate itself, namely the Kerr shutter. A schematic representation of its principle is depicted in Fig. 2. Here, the control and the signal beam, generated by two independent oscillators, are focused onto a highly NL medium. This will produce the ps-gated signal at $1 \mu \mathrm{m}$ that will be further amplified ( $3^{r d}$ block). The ns signal beam is provided by a diode-pumped solid-state Nd:YAG actively Q-switched microchip laser (STANDA-Q1) that generates $1 \mu \mathrm{m}$ ns pulses centered at $1064 \mathrm{~nm}$ with an excellent beam quality $\left(M^{2} \sim 1.15\right)$ and energy of $140 \mu \mathrm{J}$ at $1 \mathrm{kHz}$. The polarization of the control and signal pulses have to be linear and at an angle of $45^{\circ}$ to each other. The operation of the Kerr shutter can be then briefly described as follows: the control beam induces a time varying birefringence on the NL medium causing the rotation of the signal's polarization state. The use of an output polarizer, allows the selection of the ps-gated signal that has approximately the same duration as the control signal and it is optically synchronized with it due to the quasi-instantaneous character of the NL interaction.

Reliable operation of the Kerr-shutter requires both temporal and spatial overlap of the two independent sources. Regarding the temporal aspect, the initial jitter between the two beams is assured by standard electronics to be better than 100 ps rms, allowing the operation of the gate over the $1 \mu \mathrm{m}$ pulse plateau and therefore assuring low intensity variations of $<2 \%$ rms. Concerning the spatial aspect, the sizes of the control and signal beams (550 $\mu \mathrm{m}$ and $190 \mu \mathrm{m}$ diameter at $1 / e^{2}$ respectively) have been ad- equately chosen so that the following conditions are met: 1) The control pulses have sufficient intensity (operation point intensity $\sim 7.9 \mathrm{GW} / \mathrm{cm}^{2}$ ) to induce the required instantaneous dephasing of the signal pulses and 2) the overlap between the two beams is realized over the central part of the control beam to assure a spatially homogeneous dephasing over the full signal beam. Different media could be used for the NL gate. In this experiment we use a $7 \mathrm{~mm}$ thick uncoated SF14-type glass plate with a relatively high NL refractive index $\left(n_{2} \approx 10^{-19} \mathrm{~m}^{2} / W\right)$, high transparency in the NIR spectral range and low multi-photon absorption.

According to the Kerr shutter theory [15], the ps gated pulses are proportional to $\sin ^{2}\left(\Delta \phi_{N L} / 2\right)$ with $\Delta \phi_{N L}=2 \pi L / 2 n_{2} I_{\mathcal{c}}(t) \lambda_{s}$ the induced phase shift, L the length of the NL medium, $I_{C}$ the control beam's intensity and $\lambda_{s}=1064 \mathrm{~nm}$. As shown in [16], the optical gating follows well the theoretical descriptions for moderate control intensities and NL dephasing below $\sim \pi / 2$. Ideally, a $100 \%$ shutter efficiency can be obtained when a $\phi_{N L}=$ $\pi$ phase shift is achieved. However, even if possible through careful optimization of the operation point and the NL gate material, this operation regime requires intensity levels of the control pulses $\left(\sim 16.5 \mathrm{GW} / \mathrm{cm}^{2}\right)$ close to the threshold of other unwanted effects such as self-focusing or even damage of the NL medium, observed already in our setup. The $1 \mu \mathrm{m}$ beam line in our configuration is suffering of high losses due to our non-optimised optics (two isolators, TLMB mirrors, lenses, the uncoated NL medium) resulting in only $\sim 25 \%$ throughput and $\sim 35 \mu \mathrm{J}$ effective energy for the gating. We estimate the ps-gated signal at $180 \mathrm{~nJ}$ which when taking into account the ratio of the $1 \mu \mathrm{m}$ signal's initial and final pulse duration, corresponds to a maximum instantaneous gating efficiency of $\sim 52 \%$. This is in good agreement with the theoretically expected gating efficiency (47\%) for the applied control and signal intensities resulting in a $0.48 \pi$ phase shift.

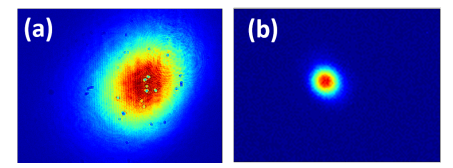

Fig. 3. Beam profile of the ps gated signal at the output of the Kerr switch in the (a) near and (b) far field.

Even if the contrast requirement for the OPCPA pump pulse is generally not strict, a high-contrast gated signal is still required so that the amplified energy is effectively concentrated in the main ps signal itself assuring also a high efficiency second harmonic generation (SHG). In the current configuration, when the Kerr shutter is closed there is still a remaining leak of the ns injection signal due to imperfections of the optical elements used, principally related to the limited extinction ratio of the polarizers. This results in a weak ns pedestal that could potentially be amplified and lead to a reduction of the gated signal's energy after amplification. Using a series of calibrated density filters and a CCD camera placed at the exit of the Kerr shutter, we could estimate the output energy ratio with the control beam blocked or not. Taking into account the pulse duration ratio for the gated and initial $1 \mu \mathrm{m}$ pulses, we were able to estimate the intensity extinction ratio at $\sim 10^{-4}$ which falls into the limitations of the commercially available high quality optical components used in our setup. According to our simulations this extinction ratio can be well tolerated by our amplifiers representing $<10 \%$ energy loss from the main ps pulses. Regarding the spatial quality of 
the ps-gated signal, it is very close to a Gaussian beam with a $M^{2} \sim 1.2$ as shown in Fig. 3.

The energy stability of the ps-gated signal is a crucial aspect of the amplifier's operation later on and it most importantly depends on the intensity stability of the control and signal beams themselves. While the signal beam is very stable $(<2 \% \mathrm{rms})$, the control suffers from an instability that can reach up to $>5.5 \%$ rms. This originates principally in the Ti:Sa amplifier pump source ( $>3 \% \mathrm{rms}$ ), air turbulence in the mostly uncovered beam path of the CPA system and thermal drifts of the non-regulated laboratory where the system is installed. The combination of these factors results in an energy stability of $\sim 7 \% \mathrm{rms}$ for the ps-gated signal which is in agreement with what is theoretically expected for a $52 \%$ gating efficiency. It is worth noting that these instabilities could be greatly attenuated if the optimal operation point of the gate, close to $100 \%$, was reached thanks to the slowly varying $\sin ^{2}\left(\Delta \phi_{N L}\right)$ function around its maximum. However, since our amplifier works in a strong saturation regime, this instability does not critically affect the performance of the overall system, and this optimization work is left for a future study.
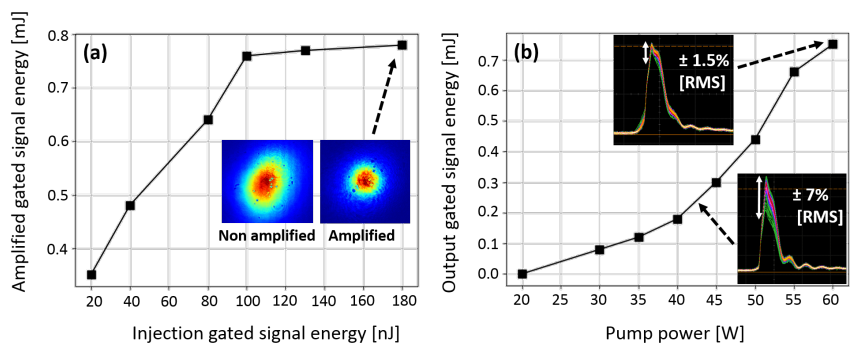

Fig. 4. Amplified ps-gated signal at the output of the $\mathrm{Nd}: \mathrm{YVO}_{4}$ amplifier (a) versus the injected ps-gated signal -as the injection energy increases the amplifier reaches saturation(b) versus the pump power showing the improvement of the output's intensity stability.

The third block consists of a single stage double-pass amplifier pumped at $808 \mathrm{~nm}$ with an $\mathrm{Nd}: \mathrm{YVO}_{4}$ crystal serving as the gain medium. The experimental setup is very similar to the one described in great detail in [17]. The amplifier is designed to deliver a total gain around $8000(39 \mathrm{~dB})$ with a maximum B-integral value estimated at $\sim 2.5$ rad when pumping with maximum power. The experimental results are in agreement with the theoretical estimations where we achieve a gain of $37 \mathrm{~dB}$ that corresponds to $>0.8 \mathrm{~mJ}$. Regarding unwanted effects such as parasitic lasing, we first evaluated the non-injected operation of the amplifier resulting in a $<60 \mu \mathrm{J}$ output. To estimate the contribution of the ns leaking pedestal of the NL gate, we measured the amplifier output in the absence of the control beam which corresponds to $\sim 100 \mu \mathrm{J}$. However, in the nominal operation of the amplifier this energy is expected to be partially suppressed by the presence of the ps-gated signal by a factor of $\sim 2 x$ and therefore presenting a small fraction of the total output energy $(<7 \%)$.

In Fig. 4 (a) we plot the output energy as a function of the energy of the injected ps-gated signal which shows that the amplifier operates in the saturation regime. The high quality ( $\sim 1.1 \times$ diffraction limit) beam profile (right side inset of Fig.4 (a)) at the amplifier's output allows us to safely assume that the Bintegral's value remains low since no beam distortion due to the Kerr lens effect is observed. The pointing stability at full
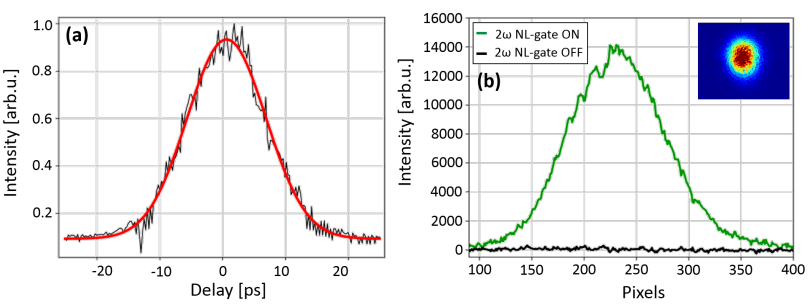

Fig. 5. (a) Autocorrelation trace of the ps-gated signal after the amplifier. Black curve: raw data, red curve: Gaussian fit. (b) Green curve: spatial profile of $532 \mathrm{~nm}$ beam, black curve: blocking the control beam leads to non-measurable signal indicating that the full SHG energy is contained in the ps pulses. Inset: beam profile of $532 \mathrm{~nm}$ beam on CCD camera.

power has been measured slightly lower than the one of the input energy at around $11 \mu \mathrm{rad}$ rms over 5 hours.

An interesting point is how the relatively low intensity stability of the ps-gated signal affects the operation of the amplifier. This is shown in Fig. 4 (b) where we plot the evolution of the output energy as a function of the pump power. As the pump power increases, one can see the intensity stability of the amplified ps-gated signal improving significantly. Around $P_{\text {pump }}=43$ $\mathrm{W}$ the signal stability is $\sim 7 \% \mathrm{rms}$ which is very similar to the non-amplified one, directly after the Kerr shutter.As the pump power increases, the signal stability improves up by a factor of 3 , reaching a rms stability of $<1.5 \%$ when pumped with maximum power. Fig. 5 (a) shows the measured autocorrelation trace and Gaussian fit of the amplified pulses at full power. The fit has a FWHM of 17 ps which indicates a pulse length of 12 ps assuming a Gaussian temporal profile.

The last step is the frequency doubling where we use a 4 mm thick type-I Lithium Triborate (LBO) crystal and focus the amplified gated beam on a $400 \mu \mathrm{m}$ spot (diameter at $1 / e^{2}$ ). For an input of $0.8 \mathrm{~mJ}$ at $1064 \mathrm{~nm}$ we obtain $>550 \mu \mathrm{J}$ at $532 \mathrm{~nm}$ corresponding to a conversion efficiency of $\sim 70 \%$. This relatively high conversion efficiency is an additional proof of both the temporal and spatial quality of the ps-gated and amplified pulses at $1 \mu \mathrm{m}$. The $2 \omega$ beam itself has an excellent spatial profile (inset Fig. 5 (b)) and an equally good intensity stability of $<2 \% \mathrm{rms}$. Furthermore, we were able to show that the measured $2 \omega$ signal is generated almost solely from the ps-gated signal. Fig. 5 (b) shows in green the measured $532 \mathrm{~nm}$ signal and when the control beam is blocked -the Kerr shutter is closed- we record almost no $2 \omega$ being generated as shown by the black curve.

For the demonstration of the compatibility of our source for ultrafast OPCPA pumping we performed a proof of principle experiment in the Apollon laser facility Front End [10]. Blocks $2 \& 3$ of Fig. 1 were mounted on a small breadboard (600x900 $\mathrm{mm}$ ) and the setup has been temporally installed in the vicinity of the actual pump system of the Apollon OPCPA source. This choice has been made to allow: 1) the demonstration of an ultrabroadband OPCPA assured by the Apollon seed signal and 2) the proof of the integration capacity of the setup in an already running system without major modifications. The details of the Apollon front end architecture are described elsewhere [10] but its current OPCPA signal beam line essentially consists in a Ti:Sa multipass amplifier that generates $1.5 \mathrm{~mJ}, 25 \mathrm{fs}$ pulses with energy stability of $<2 \%$ rms. After a cross-polarized wave (XPW) configuration, a bulk stretcher, an acousto-optic programmable dispersive filter (Dazzler) and a spatial filter the input signal 


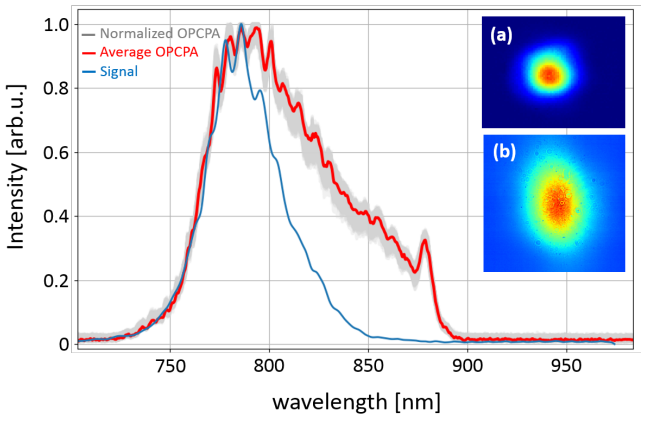

Fig. 6. Red: average OPCPA spectrum, Grey: sequence of 500 normalised OPCPA spectra, Blue: input signal. Inset: Spatial profiles on CCD camera of (a) the pump-532 nm beam on focus (b) the resulting OPCPA beam.

of the OPCPA has a maximum energy of $1 \mu \mathrm{J}$, a Gaussian like spectrum of $\sim 45 \mathrm{~nm}$ FWHM bandwidth centered at $790 \mathrm{~nm}$ and intensity stability of $3.5 \% \mathrm{rms}$. For the control beam of our Kerr shutter we insert a $10 \%$ reflective beam splitter before the compressor of the Ti:Sa multipass amplifier resulting in $200 \mu \mathrm{J}$, $\sim 15$ ps pulses on our NL gate. As the control signal in Apollon has identical characteristics with the initial setup, we were able to obtain the same operation point for the gated and amplified pulses at $1064 \mathrm{~nm}$ as described already. The $1064 \mathrm{~nm}$ pulses have been then directed in a $\sim 3 \mathrm{~m}$ delay line to balance the signal-pump optical path difference. SHG was then performed in a LBO crystal with identical characteristics as in the initial setup. Due to the limited time in the Apollon facility and the space constrains of the front end setup we have chosen to adjust the energy level rather than the beam diameters to optimize the OPCPA operation in terms of saturation level and spectral characteristics. We used therefore only about $220 \mu \mathrm{J}$ at $532 \mathrm{~nm}$, with a diameter of $\sim 400 \mu \mathrm{m}$ (Fig. 6 (a)) and a corresponding intensity of $\sim 12 \mathrm{GW} / \mathrm{cm}^{2}$. The Apollon OPCPA stage is based on a $4 \mathrm{~mm}$ thick type-I BBO crystal in a non-collinear configuration and the input signal has been attenuated to $\sim 20 \mathrm{~nJ}$ with and a 1 $\mathrm{mm}$ spot diameter on the crystal.

After optimization of the phase matching conditions we obtained $>20 \mu \mathrm{J}$ of OPCPA signal with a smooth Gaussian like beam profile (Fig. 6 (b)). This energy corresponds to $\sim 10 \%$ conversion efficiency that is typical and well compared to the nominal one for the Apollon front end. In Fig. 6 we provide the obtained averaged spectrum (red line curve) extending over $>160 \mathrm{~nm}$ along with the input one (blue line curve). The saturation of the OPCPA becomes evident by the strong amplification of the "red" side spectrum of the input beam. In the same figure we show also the accumulated normalized spectra over 1 minute corresponding to $\sim 3.4 \%$ rms energy stability (value extracted from raw spectra), principally limited by the instabilities of the seed. The evaluation of the spectral stability of the OPCPA output allows also an indirect estimation of the jitter stability between the OPCPA pump and the signal. For a temporal stretching factor of $<0.1 \mathrm{ps} / \mathrm{nm}$ and a spectral barycenter stability $<1 \mathrm{~nm}$ $(\mathrm{PtV})$ we can safely estimate the relative synchronization jitter in the sub-100 fs range. The OPCPA operation has been tested over a total time of one week and about 4 hours/day showing excellent reproducibility, both in terms of energy and spectrum with practically no need of realignment or optimization.

In conclusion, we have presented a novel configuration for the generation of optically synchronized pump-signal OPCPA pairs, originating from two independent oscillators. We demonstrated the successful operation of a Kerr shutter for the generation of energetic ps pulses from a $1 \mathrm{~ns}$ source that allowed a stable and efficient amplification in a high gain $\mathrm{Nd}: \mathrm{YVO}_{4}$ diode pumped amplifier. Frequency doubling of these pulses provided $>550 \mu \mathrm{J}$ at $532 \mathrm{~nm}$ and were later used as the pump beam in a proof of principle experiment with the ps OPCPA system of the Apollon facility front end. Given its simplicity and adaptability, our method could be easily added in already existing systems in a cost effective and non-invasive manner and could also be transferred to any couple of signal-pump wavelengths.

Funding. "Investissements d'Avenir" LabEx PALM (ANR-10LABX-0039-PALM), ILE APOLLON 07-CPER 017-01.

Disclosures. The authors declare no conflicts of interest.

\section{REFERENCES}

1. A. Piskarskas, A. Stabinis, and A. Yankauskas, Sov. Phys. Uspekhi 29, 869 (1986).

2. P. Rudawski, A. Harth, C. Guo, E. Lorek, M. Miranda, C. M. Heyl, E. W. Larsen, J. Ahrens, O. Prochnow, T. Binhammer, U. Morgner, J. Mauritsson, A. L'Huillier, and C. L. Arnold, The Eur. Phys. J. D 69 (2015).

3. M. Baudisch, B. Wolter, M. Pullen, M. Hemmer, and J. Biegert, Opt. Lett. 41, 3583 (2016).

4. C. N. Danson, C. Haefner, J. Bromage, T. Butcher, J.-C. F. Chanteloup, E. A. Chowdhury, A. Galvanauskas, L. A. Gizzi, J. Hein, D. I. Hillier, N. W. Hopps, Y. Kato, E. A. Khazanov, R. Kodama, G. Korn, R. Li, Y. Li, J. Limpert, J. Ma, C. Hee Nam, D. Neely, D. Papadopoulos, R. R. Penman, L. Qian, J. J. Rocca, A. A. Shaykin, C. W. Siders, Spindloe, Christopher, S. Szatmári, R. M. G. M. Trines, J. Zhu, P. Zhu, and J. D. Zuegel, High Power Laser Sci. Eng. 7, e54 (2019).

5. F. Lureau, G. Matras, O. Chalus, C. Derycke, T. Morbieu, C. Radier, O. Casagrande, S. Laux, S. Ricaud, G. Rey, A. Pellegrina, C. Richard, L. Boudjemaa, C. Simon-Boisson, A. Baleanu, R. Banici, A. Gradinariu, C. Caldararu, B. De Boisdeffre, P. Ghenuche, A. Naziru, G. Kolliopoulos, L. Neagu, R. Dabu, I. Dancus, and D. Ursescu, High Power Laser Sci. Eng. 8, e43 (2020).

6. A. Galvanauskas, A. Hariharan, D. Harter, M. A. Arbore, and M. M. Fejer, Opt. Lett. 23, 210 (1998).

7. C. Y. Teisset, N. Ishii, T. Fuji, T. Metzger, S. Köhler, R. Holzwarth, A. Baltuška, A. M. Zheltikov, and F. Krausz, Opt. Express 13, 6550 (2005).

8. N. Ishii, C. Y. Teisset, T. Fuji, S. Kohler, K. Schmid, L. Veisz, A. Baltuska, and F. Krausz, IEEE J. Sel. Top. Quantum Electron. 12, 173 (2006).

9. S. Prinz, M. Haefner, C. Y. Teisset, R. Bessing, K. Michel, Y. Lee, X. T. Geng, S. Kim, D. E. Kim, T. Metzger, and M. Schultze, Opt. Express 23, 1388 (2015).

10. D. N. Papadopoulos, P. Ramirez, K. Genevrier, L. Ranc, N. Lebas, A. Pellegrina, C. L. Blanc, P. Monot, L. Martin, J. P. Zou, F. Mathieu, P. Audebert, P. Georges, and F. Druon, Opt. Lett. 42, 3530 (2017).

11. P. Rigaud, A. V. de Walle, M. Hanna, N. Forget, F. Guichard, Y. Zaouter, K. Guesmi, F. Druon, and P. Georges, Opt. Express 24, 26494 (2016).

12. S. Toth, T. Stanislauskas, I. Balciunas, R. Budriunas, J. Adamonis, R. Danilevicius, K. Viskontas, D. Lengvinas, G. Veitas, D. Gadonas, A. Varanavičius, J. Csontos, T. Somoskoi, L. Toth, A. Borzsonyi, and K. Osvay, J. Physics: Photonics 2, 045003 (2020).

13. J. Suzuki, K. Taira, Y. Fukuchi, Y. Ozeki, T. Tanemura, and K. Kikuchi, Electron. Lett. 40, 445 (2004).

14. H. Purwar, S. Idlahcen, C. Rozé, D. Sedarsky, and J.-B. Blaisot, Opt. Express 22, 15778 (2014).

15. J. M. Dziedzic, R. H. Stolen, and A. Ashkin, Appl. Opt. 20, 1403 (1981).

16. C. Alexandridi, F. Lemaitre, X. Délen, F. Druon, P. Georges, and D. Papadopoulos, Laser Congr. 2019 (ASSL, LAC, LS\&C) p. JTh3A.14 (2019).

17. X. Délen, F. Balembois, and P. Georges, Opt. Lett. 39, 997 (2014). 


\section{FULL REFERENCES}

1. A. Piskarskas, A. Stabinis, and A. Yankauskas, "Phase phenomena in parametric amplifiers and generators of ultrashort light pulses," Sov. Phys. Uspekhi 29, 869-879 (1986).

2. P. Rudawski, A. Harth, C. Guo, E. Lorek, M. Miranda, C. M. Heyl, E. W. Larsen, J. Ahrens, O. Prochnow, T. Binhammer, U. Morgner, J. Mauritsson, A. L'Huillier, and C. L. Arnold, "Carrier-envelope phase dependent high-order harmonic generation with a high-repetition rate opcpa-system," The Eur. Phys. J. D 69 (2015).

3. M. Baudisch, B. Wolter, M. Pullen, M. Hemmer, and J. Biegert, "High power multi-color opcpa source with simultaneous femtosecond deepuv to mid-ir outputs," Opt. Lett. 41, 3583-3586 (2016).

4. C. N. Danson, C. Haefner, J. Bromage, T. Butcher, J.-C. F. Chanteloup, E. A. Chowdhury, A. Galvanauskas, L. A. Gizzi, J. Hein, D. I. Hillier, N. W. Hopps, Y. Kato, E. A. Khazanov, R. Kodama, G. Korn, R. Li, Y. Li, J. Limpert, J. Ma, C. Hee Nam, D. Neely, D. Papadopoulos, R. R. Penman, L. Qian, J. J. Rocca, A. A. Shaykin, C. W. Siders, Spindloe, Christopher, S. Szatmári, R. M. G. M. Trines, J. Zhu, P. Zhu, and J. D. Zuegel, "Petawatt and exawatt class lasers worldwide," High Power Laser Sci. Eng. 7, e54 (2019).

5. F. Lureau, G. Matras, O. Chalus, C. Derycke, T. Morbieu, C. Radier, O. Casagrande, S. Laux, S. Ricaud, G. Rey, A. Pellegrina, C. Richard, L. Boudjemaa, C. Simon-Boisson, A. Baleanu, R. Banici, A. Gradinariu, C. Caldararu, B. De Boisdeffre, P. Ghenuche, A. Naziru, G. Kolliopoulos, L. Neagu, R. Dabu, I. Dancus, and D. Ursescu, "High-energy hybrid femtosecond laser system demonstrating $2 \times 10$ pw capability," High Power Laser Sci. Eng. 8, e43 (2020).

6. A. Galvanauskas, A. Hariharan, D. Harter, M. A. Arbore, and M. M. Fejer, "High-energy femtosecond pulse amlification in a quasi-phasematched parametric amplifier," Opt. Lett. 23, 210-212 (1998).

7. C. Y. Teisset, N. Ishii, T. Fuji, T. Metzger, S. Köhler, R. Holzwarth, A. Baltuška, A. M. Zheltikov, and F. Krausz, "Soliton-based pump-seed synchronization for few-cycle opcpa," Opt. Express 13, 6550-6557 (2005).

8. N. Ishii, C. Y. Teisset, T. Fuji, S. Kohler, K. Schmid, L. Veisz, A. Baltuska, and F. Krausz, "Seeding of an eleven femtosecond optical parametric chirped pulse amplifier and its nd/sup 3+/ picosecond pump laser from a single broadband ti:sapphire oscillator," IEEE J. Sel. Top. Quantum Electron. 12, 173-180 (2006).

9. S. Prinz, M. Haefner, C. Y. Teisset, R. Bessing, K. Michel, Y. Lee, X. T. Geng, S. Kim, D. E. Kim, T. Metzger, and M. Schultze, "Cep-stable, sub-6 fs, 300-khz opcpa system with more than $15 \mathrm{w}$ of average power," Opt. Express 23, 1388-1394 (2015).

10. D. N. Papadopoulos, P. Ramirez, K. Genevrier, L. Ranc, N. Lebas, A. Pellegrina, C. L. Blanc, P. Monot, L. Martin, J. P. Zou, F. Mathieu, P. Audebert, P. Georges, and F. Druon, "High-contrast 10 fs opcpabased front end for multi-pw laser chains," Opt. Lett. 42, 3530-3533 (2017).

11. P. Rigaud, A. V. de Walle, M. Hanna, N. Forget, F. Guichard, Y. Zaouter, K. Guesmi, F. Druon, and P. Georges, "Supercontinuum-seeded fewcycle mid-infrared opcpa system," Opt. Express 24, 26494-26502 (2016).

12. S. Toth, T. Stanislauskas, I. Balciunas, R. Budriunas, J. Adamonis, R. Danilevicius, K. Viskontas, D. Lengvinas, G. Veitas, D. Gadonas, A. Varanavičius, J. Csontos, T. Somoskoi, L. Toth, A. Borzsonyi, and K. Osvay, "SYLOS lasers - the frontier of few-cycle, multi-TW, $\mathrm{kHz}$ lasers for ultrafast applications at extreme light infrastructure attosecond light pulse source," J. Physics: Photonics 2, 045003 (2020).

13. J. Suzuki, K. Taira, Y. Fukuchi, Y. Ozeki, T. Tanemura, and K. Kikuchi, "All-optical time-division add-drop multiplexer using optical fibre kerr shutter," Electron. Lett. 40, 445-446 (2004).

14. H. Purwar, S. Idlahcen, C. Rozé, D. Sedarsky, and J.-B. Blaisot, "Collinear, two-color optical kerr effect shutter for ultrafast time-resolved imaging," Opt. Express 22, 15778-15790 (2014).

15. J. M. Dziedzic, R. H. Stolen, and A. Ashkin, "Optical kerr effect in long fibers," Appl. Opt. 20, 1403-1406 (1981).

16. C. Alexandridi, F. Lemaitre, X. Délen, F. Druon, P. Georges, and D. Papadopoulos, "Kerr shutter for the generation of optically synchronized pump-signal opcpa pairs," Laser Congr. 2019 (ASSL, LAC, LS\&C) p. JTh3A.14 (2019).

17. X. Délen, F. Balembois, and P. Georges, "Direct amplification of a nanosecond laser diode in a high gain diode-pumped nd:yvo4 amplifier," Opt. Lett. 39, 997-1000 (2014). 